\title{
Shaping Resonant Light Confinement and Optoelectronic Spectra Using Strain in Hierarchical Multiscale Structures
}

\author{
Jagdish Anakkavoor Krishnaswamy, Kumar Molahalli Panidhara, Sandeep B. Satyanarayana, \\ Praveen Chandrashekarapura Ramamurthy, * Debiprosad Roy Mahapatra, \\ and Gopalkrishna Hegde
}

\begin{abstract}
Hierarchically structured optical materials have been a topic of intriguing research interest due to the possibility of tailoring material properties beyond the limits of bulk continuum material design. Here, an optical confinement phenomenon in a hierarchically structured waveguide platform consisting of alternating clusters of nanostructured and planar microscale domains is demonstrated. An unconventional self-assembly-based strain-assisted nanomolding process is developed to fabricate these hierarchically structured multiperiodic waveguides. Further, these hierarchically patterned waveguides are used as substrates for solution-processed photodetectors. The optical confinement occurring due to the nanoscale scattering and the wavelengthdependent interaction between the planar and structured microdomains leads to an improved uniformity in the optoelectronic spectral response of these photodetectors. Furthermore, by tuning the multiperiodicity within the nanostructured domain structure, using a mechanical strain of $20 \%$, an improvement of around $10 \%$ in the uniformity of the optoelectronic spectral response of the photodetectors is demonstrated. Simulations further show that these processes arise only in the presence of a hierarchical structure, due to multiscale interaction, through wavelength-selective coupling of scattered light from nanostructured domains to the planar microdomains. In summary, hierarchical structures can address optoelectronic problems that cannot be addressed by simpler structures.
\end{abstract}

\section{Introduction}

Hierarchical structuring in natural and man-made materials has given rise to interesting design pathways that extend the material properties beyond the continuum regime ${ }^{[1]}$ and incorporate multiple functionalities on the same macrostructural platform. ${ }^{[2]}$ The idea consists of designing structural components that themselves are structured at multiple levels, through the introduction of features at multiple length scales. Notable consequences of multiscale design include simultaneous enhancement of strength and toughness, ${ }^{[1,3]}$ counterintuitive behavior such as negative Poisson's ratios, ${ }^{[1,4]}$ biological examples of integrating multiple functions such as antireflection, superhydrophobicity, and antifogging in the eyes of insects, ${ }^{[2,5,6]}$ tuning mechanical behavior of layered materials by hierarchical structuring while retaining macroscale electrical conductivity, ${ }^{[7]}$ and several others. Few studies have demonstrated that a hierarchical pattern is superior to a single-level pattern in terms of superhydrophobicity ${ }^{[8]}$ and light trapping for enhanced absorption in solar cells. ${ }^{[9]}$ Hierarchically structured polymeric materials have interesting implications in biomedical applications owing to the possibility of precise and programmed drug delivery and efficient tissue scaffolding due to large surface areas. ${ }^{[10]}$ In the area of energy storage, electrode materials with multiscale structures enable simultaneously aspects such as enhanced battery performance and better structural stability, by taking advantage of sizedependent material properties. ${ }^{[11,12]}$ The efficacy of multiscale design is also evident from ecological studies that show that forests with a diverse flora spanning a range of heights, and hence constituting a multiscale canopy, lead to a very efficient interception of sunlight, thus offering another biological motivation to hierarchical structuring. ${ }^{[13]}$ In the area of optoelectronic design, however, most of the literature till date is focused on the effects of a single level of patterning such as continuous nanostructured arrays for light confinement in the context of solid-state photovoltaics, ${ }^{[14-23]}$ to maximize the absorption of light per unit device area. The main goal of this design is to obtain a complete nanostructural coverage over the device area to maximize light

DOI: 10.1002/adom.201900471 
trapping and absorption. In other words, the inherent goal of material design is to synthesize defect-free materials and structures to maximize a desired optoelectronic function. However, such an approach often overlooks rich multiscale photonic processes occurring in a hierarchical structure. For example, a defective nanostructured array with microscale discontinuities, which is a hierarchical structure with two length scales, can exhibit optical transport characteristics that are totally different from the defect-free constituent nanostructured array and the planar (continuum) structures. There have been some investigations carried out concerning the relevance of multiscale/hierarchical designing in photonics and optoelectronics. These include using hierarchically patterned photovoltaic architectures to boost photocurrents beyond the limits of a single-level pattern, $[9,24,25]$ design of fractal-like nanostructures for multimodal plasmonic resonance to broaden spectral response, ${ }^{[26]}$ hybrid nanostructures for efficient photodetectors, ${ }^{[27]}$ and energy harvesters for self-powered nanostructured photodetectors. ${ }^{[28]}$ Particularly, the design advantages of hierarchically structured electrodes have been demonstrated in the area of dye-sensitized solar cells. The research in this area has shown that hierarchical structures offer multiple simultaneous advantages, which include light trapping and absorption enhancement, higher surface area and better charge collection, and better electrolyte diffusion, ${ }^{[29,30]}$ demonstrating the advantage in terms of photovoltaic enhancement. However, outside the purview of the well-known challenges of photovoltaic/photodetecting absorption enhancement, these investigations are at an early stage of exploratory investigation where the implications of hierarchical structuring in optoelectronics are yet to be explored. We emphasize that the hierarchical structures can offer solutions to many other problems that cannot be addressed using simpler nanostructures. This is the central aspect of this paper.

In the process of investigating hierarchical multiscale structures, we also address another important aspect of nanofabrication-to extend soft lithographic and nanoimprint lithographic fabrication beyond the limits of the monoperiodic geometric features of the master template. Traditionally, these processes use a nanostructured master mold or template, the features of which are replicated on the substrate of interest. Soft lithography has been extensively investigated to form nanostructures by using moldable elastomers. ${ }^{[31]}$ This fabrication process is typically based on self-assembled periodic monolayers of nano- or microsized spherical particles. ${ }^{[32-35]}$ However, the structures developed using this method are monoperiodic with the periodicity limited by the diameter of the sphere. Nanoimprint lithography also uses a mold to deform a temporary film that is deposited on the target material to be patterned. ${ }^{[36]}$ Investigations have revealed several interesting phenomena that can be tuned to control the surface wetting behavior, ${ }^{[37]}$ optical behavior of the structure, ${ }^{[38]}$ and so on. However, a majority of these investigations use the nanostructures that are just replicas of the features in the master template. This paper also addresses the question whether the limits of nanoscale fabrication using the molded templates can be extended, through the development of unconventional but facile fabrication processes, to obtain feature sizes that are either larger or smaller than those in the master template. The bigger question here is whether the tuning of feature sizes can lead to control over multiscale optical transport. This is the second aspect of this paper.
In terms of physical phenomena, we are concerned with the complex optical transport that occurs in the two-scale hierarchical structure-one is at the superwavelength scale and the other is at wavelength scale. We demonstrate that the resonant coupling of light among the superwavelength features (for $n=2$, Figure 1c) leads to spectral signatures that are observable neither in a material continuum $(n=0$, Figure $1 \mathrm{a})$ nor in a perfectly crystalline ( $n=1$, Figure $1 \mathrm{~b}$ ) architecture, where $n$ is the order of hierarchical structuring. This becomes possible because of the coexistence of dual functionalities in the twoscale hierarchical structure for $n=2$ (Figure 1c), light scattering by the wavelength-scale structures, and resonant coupling across the larger domains.

Further, we have studied the practical implications of such multiscale photonic design, and we have used the underlying phenomenon to tune the optoelectronic response of a solutionprocessed photodetector built on the hierarchically structured platforms, and to consequently improve the spectral uniformity of the optoelectronic response of the device. Optoelectronic spectral shaping is an important factor in the design of photodetectors. Nonuniform spectral intensities are inevitable in thin-film optoelectronic devices that heavily rely on the use of high-refractive-index transparent oxide electrodes or buffer layers such as indium tin oxide, fluorine-doped tin oxide, aluminum-doped zinc oxide, and $\mathrm{TiO}_{2}$, although their macroarchitectures for thin-film optoelectronic devices have been studied extensively. ${ }^{[39-42]}$ These unwanted nonuniformities in the spectral features are due to the strong optical contrast between the constituent layers of the device. A planar device structure based on a $\mathrm{TiO}_{2}$ film shows a strong photoresponse around $\lambda=400 \mathrm{~nm}$, as seen from the simulations in Figure $1 \mathrm{~d}$. This response is a signature of the optical interfaces in the device architecture, which cannot be suppressed by trivial manipulation of the layer thicknesses (see Section S1 in the Supporting Information). These spectral features tend to reduce the spectral range and uniformity of the photoresponse, which are important for practical applications. ${ }^{[43]}$ There have been some efforts to tune the spectral response of photodetectors by introducing a resonant optical cavity in the device. ${ }^{[4-46]}$ However, this design strategy introduces optical elements in the device path, and the optical design might interfere with the electrical performance of the device; hence, it requires a careful optimization of materials and device geometry. Thus, a good design would spatially decouple the passive optical elements and the electrical function of the device. We adopt this design rule by incorporating a multiscale pattern in the substrate, consisting of alternating nanostructured and planar domains as shown in Figure 1c, on which photodetectors are fabricated. As seen in Figure 1e, this device structure converts the high-index oxide layer into a multiscale waveguide. We expect the nanostructured domain to scatter light into the waveguide, and that this light will be coupled across to the microscale planar domains, in a wavelength-dependent manner. By doing this, we incorporate an optical filtering function before the light is absorbed by the semiconducting layer, which will possibly improve the uniformity of the spectral response. In fact, the inset in Figure 1d shows a $\mathrm{TiO}_{2}$ multiscale waveguide illuminated by a normally incident $650 \mathrm{~nm}$ laser spot. It is clearly seen that the lateral optical transport across the substrate illuminates the entire substrate, hinting significantly large 


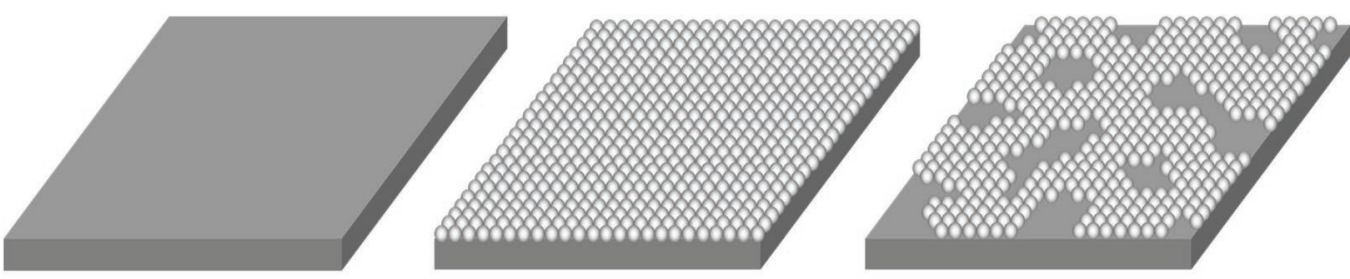

(a) $n=0$

(b) $n=1$

(c) $n=2$

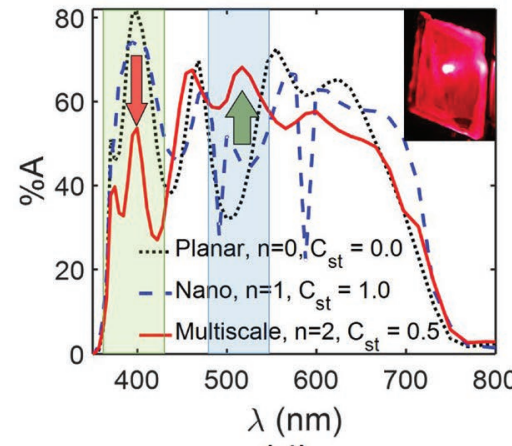

(d)

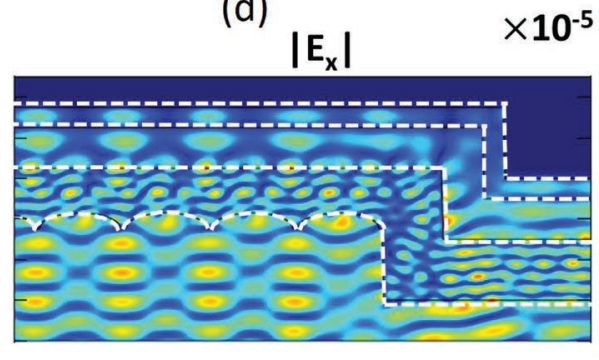

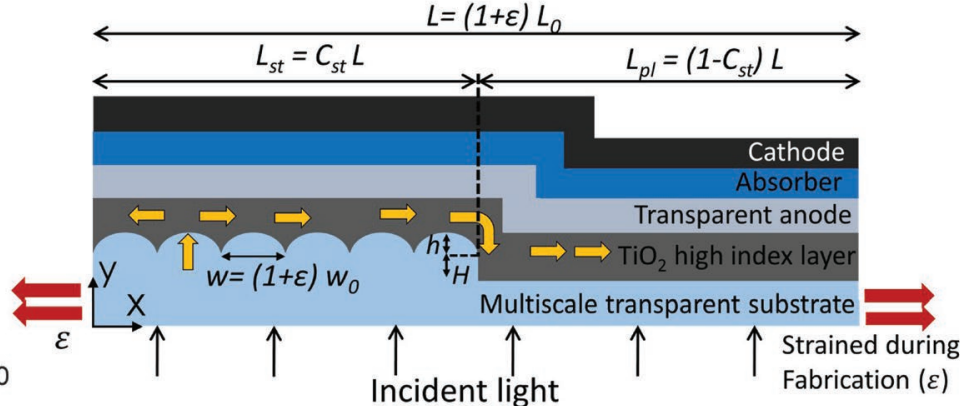

(e)

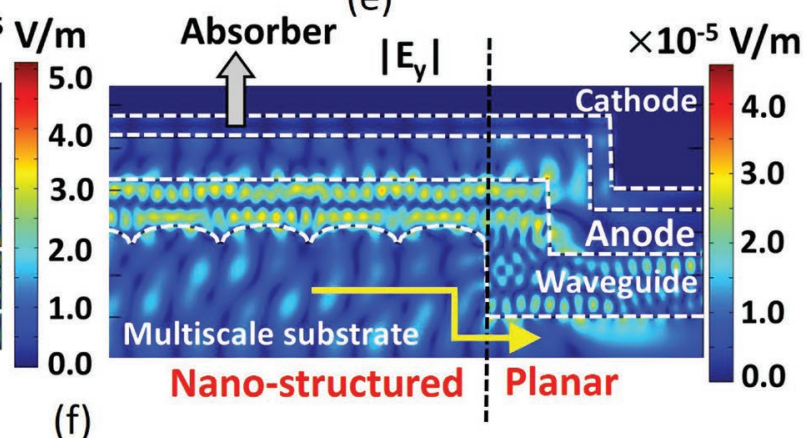

Figure 1. a-c) Schematic illustration of the hierarchical patterns, where $n$ is the hierarchical level (the inset in (c) shows a TiO ${ }_{2}$ waveguide built on a hierarchically structured substrate; the normally incident $650 \mathrm{~nm}$ laser spot at the center illuminates the whole substrate due to lateral optical transport). d) Simulated absorptance spectra of photodetectors based on planar, completely nanostructured $\left(C_{\mathrm{st}}=1.0\right)$, and partially structured $\left(C_{\mathrm{st}}=0.5\right)$ devices. e) A 2D schematic of the multiscale pattern with the illustration of the coupling of scattered light from the nanostructured to the planar microdomain, $C_{\mathrm{st}}$ is the nanostructured areal coverage, and $\varepsilon$ is the tensile strain applied on the mold during fabrication of the substrate. f) The electric field patterns for $\lambda=400 \mathrm{~nm}$ showing the cross-domain light coupling and confinement of light in the dielectric oxide waveguide.

(at least of the order of few millimeters) photonic diffusion lengths within the waveguide. This hierarchical pattern is characterized here by a parameter $C_{\mathrm{st}}$, which is the fraction of the substrate area over which there is nanostructured cover. This architecture selectively confines light within the high-index oxide layer through wavelength-dependent coupling of scattered light from the structured domain to the planar domain. Indeed, the simulated electric field patterns in Figure 1f, for $\lambda=400 \mathrm{~nm}$, clearly show guided modes with electric fields having both $x$ and $y$ components, while the incident light consists only of an electric field along the $x$-direction, proving the presence of multiscale guided modes. As seen from the simulated absorptance spectra in Figure 1d, for a hierarchical structure with $C_{\mathrm{st}}=$ 0.5 , the modified design simultaneously suppresses the peak at $\lambda=400 \mathrm{~nm}$ and improves the spectral response around $\lambda=$ $500 \mathrm{~nm}$, ultimately resulting in an improved spectral range and more uniformity of the spectral intensities. This behavior cannot be realized with a periodic nanostructured substrate without the interspersed planar microdomains (as seen in Figure 1d, from the spectrum corresponding to $C_{\mathrm{st}}=1.0$, of a defect-free continuous nanostructured array) implying that a multiscale photonic transport process is at the core of the observed behavior. In the following sections, we discuss on the details of simulations and experiments that we used to design, fabricate, and evaluate the solution-processed photodetectors on the multiscale patterned substrates. These monolithic substrates were fabricated by soft lithography on moldable thermoset epoxies. Further, we developed a novel mechanical-strain-aided molding process that enables tuning the size of the nanostructures while working with the same master template, to obtain multiperiodic nanostructures that enable tuning the spectral response of the photodetector.

\section{Results and Discussion}

\subsection{Shaping Light Confinement}

Prior to fabricating the device, we characterized the multiscale optical confinement combined with waveguiding phenomena 


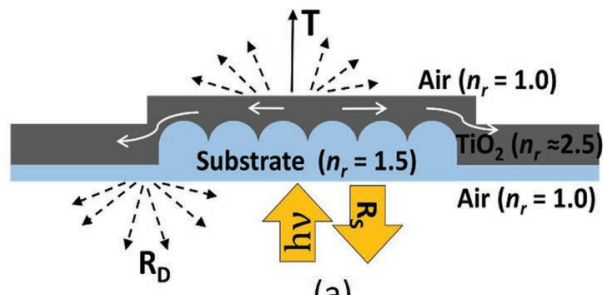

(a)

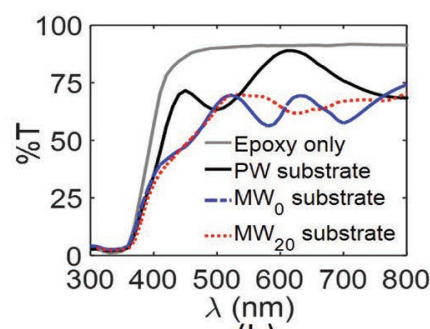

(b)

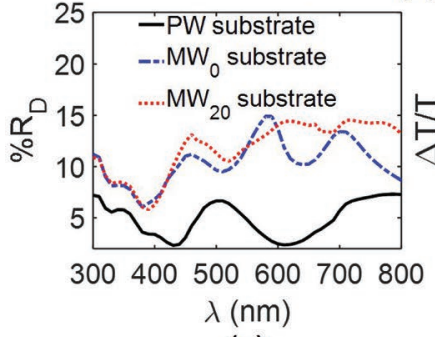

(c)

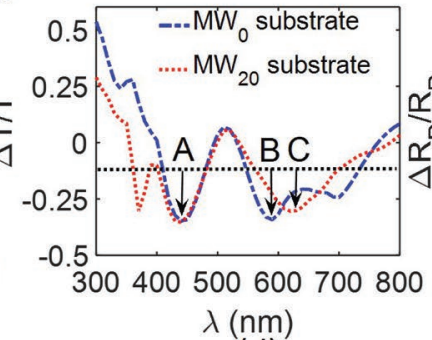

(d)

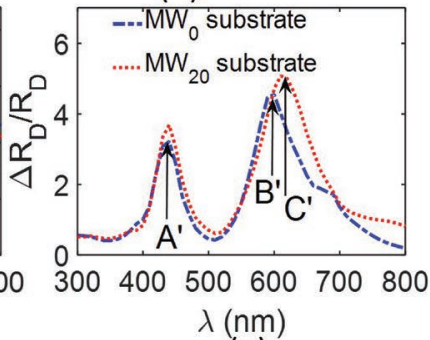

(e)

Figure 2. a) Schematic showing the device test condition for optical characterization. b) The total transmittance of the bare epoxy, PW substrate with epoxy $/ \mathrm{TiO}_{2}$ layers, and $\mathrm{MW}_{\varepsilon}$ substrate with strain $\varepsilon$ used in molding. c) Diffuse reflectance of the PW and $\mathrm{MW} \mathrm{W}_{\varepsilon}$ with varying strain during fabrication. d) Relative change in the transmittance of the $\mathrm{MW}_{\varepsilon}$ substrates with respect to that of PW substrates. e) Relative change in the diffuse reflectance of the $\mathrm{MW}_{\varepsilon}$ substrates with respect to that of PW substrates.

in the substrate (Figure 2a). Light entering the substrate will be scattered by the nanostructured domains and will get coupled to the waveguide modes in the substrate as shown schematically in Figure 2a. This is further evident from a decreased specular reflectance in the multiscale waveguide $\left(\mathrm{MW}_{\varepsilon}\right)$ substrates compared to the planar waveguide (PW) substrates (Section S4, Supporting Information). Also, the bare epoxy substrates without the $\mathrm{TiO}_{2}$ layer show no differences in the spectral transmittance (Section S4, Supporting Information), which implies that confinement of light within the $\mathrm{TiO}_{2}$ layered waveguide leads to the observed differences in the spectral behavior. The light that is not confined is transmitted out of the opposite side of the substrate constituting the total transmittance $T$ and the confined light constitutes the diffuse reflectance $R_{\mathrm{D}}$. The transmittance of the planar substrate shows a peak around $\lambda=450 \mathrm{~nm}$ and a large dip following this peak, with a local minimum around $\lambda=500 \mathrm{~nm}$. As discussed in Figure $1 \mathrm{~d}$, this peak is a characteristic feature of the planar interfaces in the substrate and cannot be suppressed by merely tuning the thicknesses of the oxide layer. However, the $\mathrm{MW}_{0}$ and $\mathrm{MW}_{20}$ substrates show a marked reduction in transmittance by almost $30 \%$ for $\lambda=450 \mathrm{~nm}$ (Figure $2 \mathrm{~b}$ ). It is important to note here that the nanostructured coverage $C_{\text {st }}$ of around $50 \%$ was used in these experiments. The primary design goal in deciding the optimum coverage was to suppress the spectral features around $\lambda=400 \mathrm{~nm}$. It was seen through transmittance measurements that higher coverages lead to a greater suppression of this spectral feature (Section S4, Supporting Information). With the spin coating process used here, the maximum uniform coverage possible was 54\%. Higher coverages were still possible but at the cost of reduced lateral uniformity and thus we fixed the optimum coverage to $54 \%$ for our study. It is interesting to note that the relative drop in the transmittance (dips $A, B$, and C in Figure 2d) and the relative increase in the diffuse reflectance $R_{\mathrm{D}}$ of the $\mathrm{MW}_{\varepsilon}$ substrate (peaks $\mathrm{A}^{\prime}, \mathrm{B}^{\prime}$, and $\mathrm{C}^{\prime}$ in
Figure 2e) show excellent agreement in terms of the spectral peak positions and spectral shifts as a function of $\varepsilon$. Therefore, it is possible to tune the transmittance of the $\mathrm{MW}_{\varepsilon}$ substrate through the tuning of the diffuse reflectance that arises from scattered light leaving the substrate. Thus, by controlling the strain, $\varepsilon$, applied on the polydimethylsiloxane (PDMS) mold, it is possible to tune the spectral dependence of the scattering in the substrate, and to induce spectral shifts such as the shift of peak $B^{\prime}$ to peak $C^{\prime}$ in Figure 2e. This provides a way to tune the transmittance of the substrate by controlling a process parameter on an intermediate molding step, while still retaining the same master template. This observation further suggests that optical confinement, which is the key process underlying spectral behavior, can be shaped using mechanical strain as a fabrication process parameter. In these optical measurements, the optical interface on either side of the substrate is with air $\left(n_{\mathrm{r}}=1\right)$. However, in an actual device, a low-index anode $\left(n_{\mathrm{r}} \approx 1.5\right)$ is deposited on the waveguide. This will slightly modify the waveguiding characteristics. However, based on the simulated absorptances of the devices (Figure 1d), the drop in the absorptance at $\lambda=400 \mathrm{~nm}$ still exists, and Figure $1 \mathrm{f}$ further suggests that this is due to lateral multiscale coupling of light. Motivated by this, we further fabricate photodetectors on the multiscale waveguides and discuss the results in the following section.

\subsection{Optoelectronic Spectral Tuning by Resonant Light Coupling}

The PW and $\mathrm{MW}_{\varepsilon}$ substrates are incorporated in a solutionprocessed photodetector. As suggested by the schematic in Figure 3a, we are interested to know whether the multiscale $\mathrm{MW}_{\varepsilon}$ substrates can filter the light entering the absorber, through multiscale optical coupling, and improve the uniformity of the optoelectronic spectral response. 


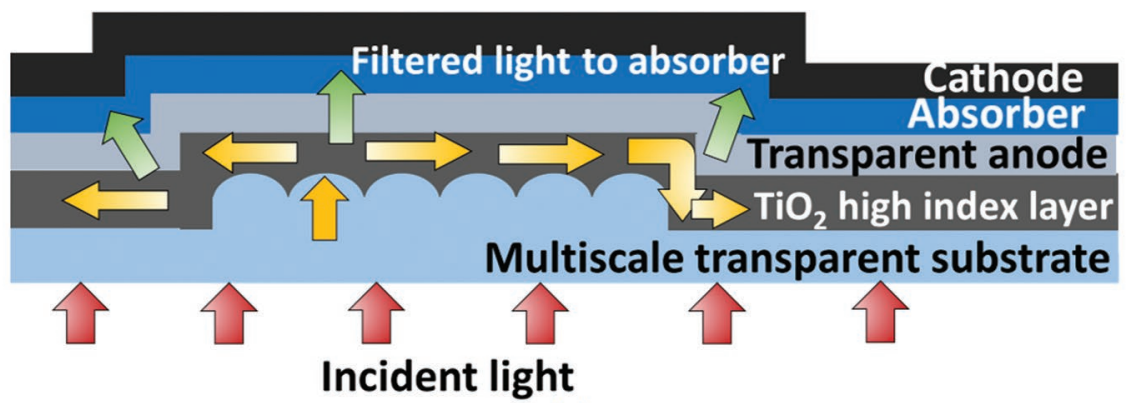

(a)
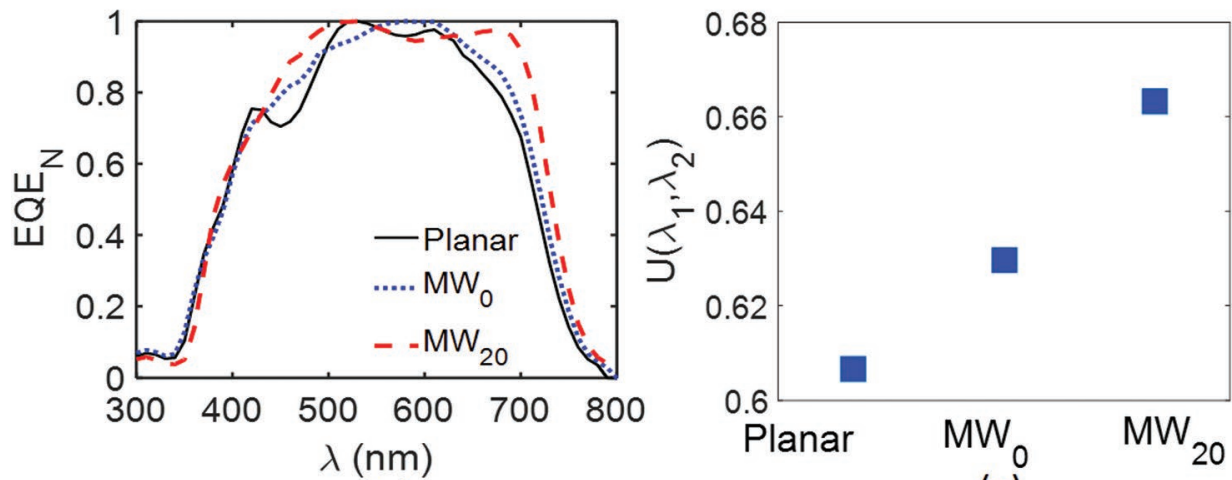

(b)

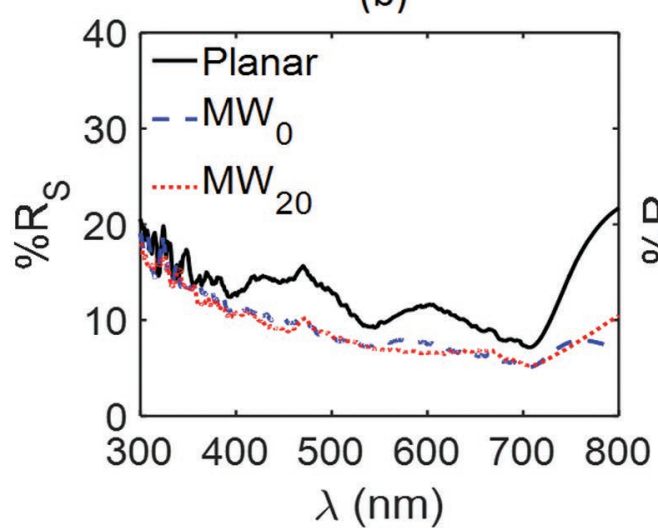

(d)

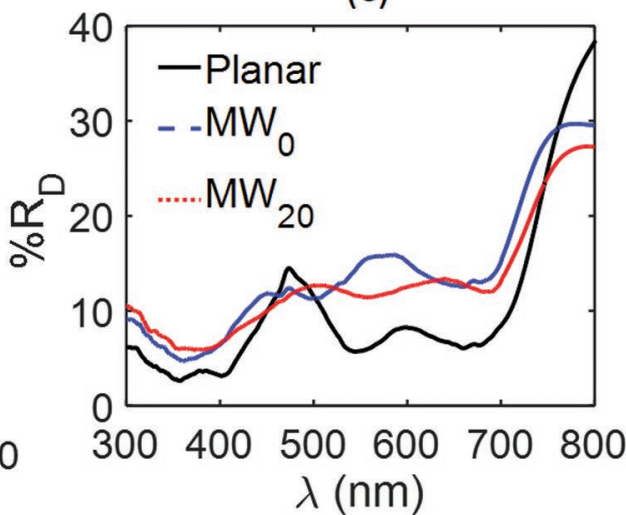

(e)

Figure 3. a) Schematic of the photodetector built on a multiscale hierarchically structured substrate and its optical working. b) The scaled EQE spectra, c) the spectral uniformity $U\left(\lambda_{1}, \lambda_{2}\right)$, d) the specular reflectance $R_{S}$, and e) the diffuse reflectance $R_{\mathrm{D}}$ of the devices based on the PW, MW, and MW $\mathrm{N}_{20}$ substrates.

Optoelectronic characterization of the devices is carried out through the measurement of $I-V$ curves, and more importantly, through the measurement of the external quantum efficiency (EQE), which provides the optoelectronic spectral signature of the devices. The spectral response of the photodetectors is analyzed in terms of its uniformity, $U$, within the wavelength range $\left(\lambda_{1}, \lambda_{2}\right)$ of interest, which is calculated as

$U\left(\lambda_{1}, \lambda_{2}\right)=\frac{\mathrm{EQE}_{\max }\left(\lambda_{1}, \lambda_{2}\right)}{\int_{\lambda_{1}}^{\lambda_{2}} \operatorname{EQE}(\lambda) \mathrm{d} \lambda}$

where $\lambda_{1}$ and $\lambda_{2}$ are typically the wavelengths marking the edges of the spectrum, beyond which the photodetector shows negligible response, and $\operatorname{EQE}_{\max }\left(\lambda_{1}, \lambda_{2}\right)$ is the maximum value of $\operatorname{EQE}(\lambda)$ in the wavelength range $\left(\lambda_{1}, \lambda_{2}\right)$. This definition of spectral uniformity, $U$, ensures that the closer the maximum value of the EQE to the average value, the higher the uniformity of the spectral response.

The normalized external quantum efficiency $\mathrm{EQE}_{\mathrm{N}}$ values of the devices based on the PW, $\mathrm{MW}_{0}$, and $\mathrm{MW}_{20}$ substrates are shown in Figure 3b. The measured EQE spectra and the details of the calculation of the normalized quantum efficiency from the measured quantum efficiency are provided in Section S7 (Supporting Information). On analyzing the EQE of the reference device and the devices fabricated on the $\mathrm{MW}_{\varepsilon}$ substrates, we made the following observations. First, the planar device shows a peak in the EQE around $\lambda=400 \mathrm{~nm}$, followed by a dip in the spectrum around $\lambda=450 \mathrm{~nm}$. This spectral feature, which resulted due to the planar or layered optical interfaces as discussed earlier, limits the spectral uniformity of the spectral response of the planar device. However, when an $\mathrm{MW}_{0}$ 
substrate is introduced in place of the PW substrate, the peak at $\lambda=400 \mathrm{~nm}$ and the dip at $\lambda=450 \mathrm{~nm}$ are suppressed. This results in a roughly $4 \%$ improvement in the uniformity $U$ (Figure 3c). However, the uniformity of the EQE spectrum can be further improved by using a multiscale substrate fabricated using a strained mold. In particular, the $\mathrm{MW}_{20}$ substrate shows an improvement of $\approx 9.3 \%$ in the uniformity $U$, compared to the PW substrate. The spectral range for these calculations is $\left(\lambda_{1}, \lambda_{2}\right)=(300 \mathrm{~nm}, 800 \mathrm{~nm})$. This range is chosen in accordance with the absorption spectrum of the active material of the device, and hence the EQE is negligible outside this range. Although the improvements in the spectral uniformity are not very large, it is important to note that this experiment demonstrates an example of how multiscale optical transport can be used to shape optoelectronic spectra. Through further optimization of the complex multiscale structure, better improvements could be possible. The nonuniform spectral features in the PW substrate are because of the presence of two dielectric interfaces with high contrast on either side of the $\mathrm{TiO}_{2}$ layer (the substrate with a refractive index of 1.5 on the bottom and the PH1000 anode on the other side having low refractive index of around 1.5, sandwiching the $\mathrm{TiO}_{2}$ layer having a relatively higher index of around 2.5). However, the $\mathrm{MW}_{\varepsilon}$ substrates are clearly able to mitigate the effect of dielectric mismatch on the planar interfaces of PW substrate, which is by wavelengthselective multiscale coupling of light into the $\mathrm{TiO}_{2}$ layered multiscale waveguide. The specular reflectance $R_{\mathrm{S}}$ (Figure $3 \mathrm{~d}$ ) of the device further shows that the $\mathrm{MW}_{\varepsilon}$ substrate reflects a relatively lesser light across the spectral band of interest $\left(\lambda_{1}=300 \mathrm{~nm}\right.$ to $\lambda_{2}=800 \mathrm{~nm}$ ), implying a broadband coupling of incident light to the waveguide modes in the MW substrates, thus offering experimental proof to the multiscale photonic coupling process.

The origin of the improved uniformity in the optoelectronic spectral response in the devices based on the $\mathrm{MW}_{\varepsilon}$ substrates can be understood by analyzing the diffuse reflectance $R_{\mathrm{D}}$ (Figure 3e). The peak of $R_{\mathrm{D}}$ at roughly $\lambda=475 \mathrm{~nm}$, for the device incorporating PW substrate, contributes to a dip in the EQE around this wavelength. On the other hand, the devices incorporating $\mathrm{MW}_{\varepsilon}$ substrates show a more uniform $R_{\mathrm{D}}$, without any sharp peak around $\lambda=475 \mathrm{~nm}$. A slight increase in the $R_{\mathrm{D}}$ around this band contributes to a small dip in the EQE of the device incorporating $\mathrm{MW}_{20}$ around $\lambda=570 \mathrm{~nm}$, thus showing that the light confinement within the multiscale waveguide substrate can be controlled by introduction of discontinuous multiperiodic 2D array structures, which can be tweaked through application of mechanical strain during fabrication. The improved spectral uniformity, through coupling with in-plane waveguide modes (schematically suggested in Figure 3a), however, comes at the cost of a reduced total transmittance of the substrate (Figure $6 \mathrm{~b}$ ) and therefore a reduced photocurrent from the active layer with electrodes.

\subsection{Simulation}

\subsubsection{Resonant Light Confinement across Multiscale Structures}

To understand the origin of the experimental results and observations, we simulate the optical transport behavior of the devices. For a simplistic computational study, the simulation geometry is approximated as a 2D configuration as shown in Figure 1e. A unit microcell, $42 \mu \mathrm{m}$ long, consisting of a linear array of 50 nanostructured elements placed alongside the planar domains of equal length on both sides of the unit cell, is constructed. This gives a periodically repeating microcell that is half covered $\left(C_{\mathrm{st}}=0.5\right)$ by the nanoscale features, which is made similar to the experimentally observed coverage area of the nanoscale features (Section S5, Supporting Information). The width of the nanostructured element is $w=w_{0}(1+\varepsilon)$, where $w_{0}$ is the nanostructured periodicity in the master template and $\varepsilon$ is the strain applied on the PDMS mold, during fabrication of the substrates. The 2D geometry does not model the compression in the features that occurs normal to the direction of the applied strain. Simulations are carried out in the frequency domain using finite-element-based modeling scheme in COMSOL Multiphysics software. The truncated 2D unit microcell adopted here is a simplified version that does not take into account the random distribution of the microscale planar and nanostructured clusters and thus it may not exactly model the experimental conditions. However, the construction and simulation of an exact 3D model, with conformally coated film layers, is highly complicated and computationally intensive. Therefore, the 2D model used here is sufficient to predict the nature of optical transport occurring due to a discontinuous nanostructured array. Exact simulations of the random architecture are needed for detailed engineering design of devices, which is out of the purview of this paper. Here, our attempts are focused on understanding new physical processes that may lead to novel design. More details about the simulation setup, including the governing equations, boundary conditions, and meshing configurations, are provided in Section S6 (Supporting Information). The optical transport behavior of the device structures is studied in response to an in-plane wave incident on the input boundary, and hence for the incident wave, the electric field components $E_{y}$ and $E_{z}$ are zero and only $E_{x}$ is nonzero. The results in Figure $4 \mathrm{a}-\mathrm{c}$ correspond to $\varepsilon=0$, i.e., the periodicity of the nanoscale pattern in $400 \mathrm{~nm}$, as in the master template.

The primary resonant peaks in the spectra of the planar waveguide-based architectures are shaped differently with the help of nanoscale features and further due to shape change in the nanoscale features due to application of strain in the mold for the substrate. Combined with these effects are the intermittent discontinuities of the nanoscale feature array with planar substrate domains, thereby creating a multiscale interaction. If there were no interactions between the planar domain and the nanostructured domains, the absorptance of the photodetectors can be estimated by simply averaging the absorptance of the planar $(n=0)$ and the completely covered nanostructured $(n=1)$ architectures, weighted by the areal densities (in this case of $C_{\mathrm{st}}=0.5$, the weights are 0.5 ) of each domain. That is, in the absence of multiscale optical interactions, the absorptance of the device multiscale device, with equal areal cover of nanostructures and planar domains (i.e., $C_{\mathrm{st}}=0.5$ ), would be given by

$A_{\text {superposed }}=\frac{1}{2}\left(A_{n=0}+A_{n=1}\right)$

where $A_{\text {superposed }}$ is the absorptance of the multiscale device that is given by the average of the absorptance $A_{n=0}$ of the planar 


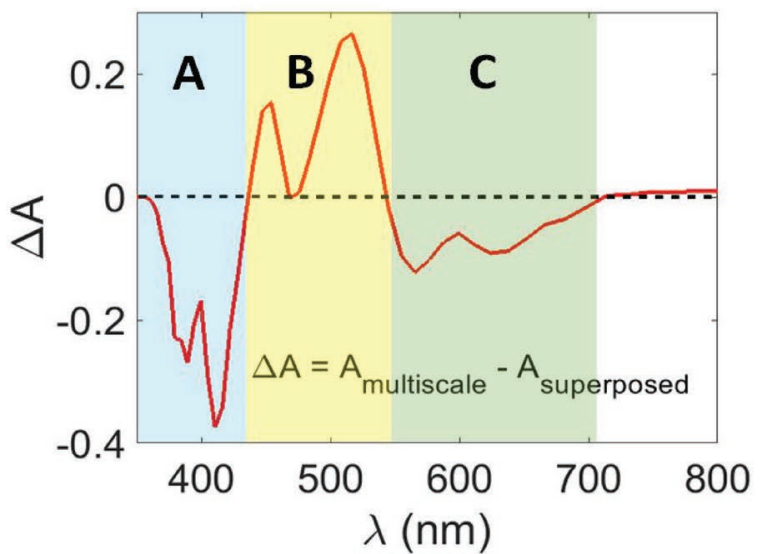

(a)
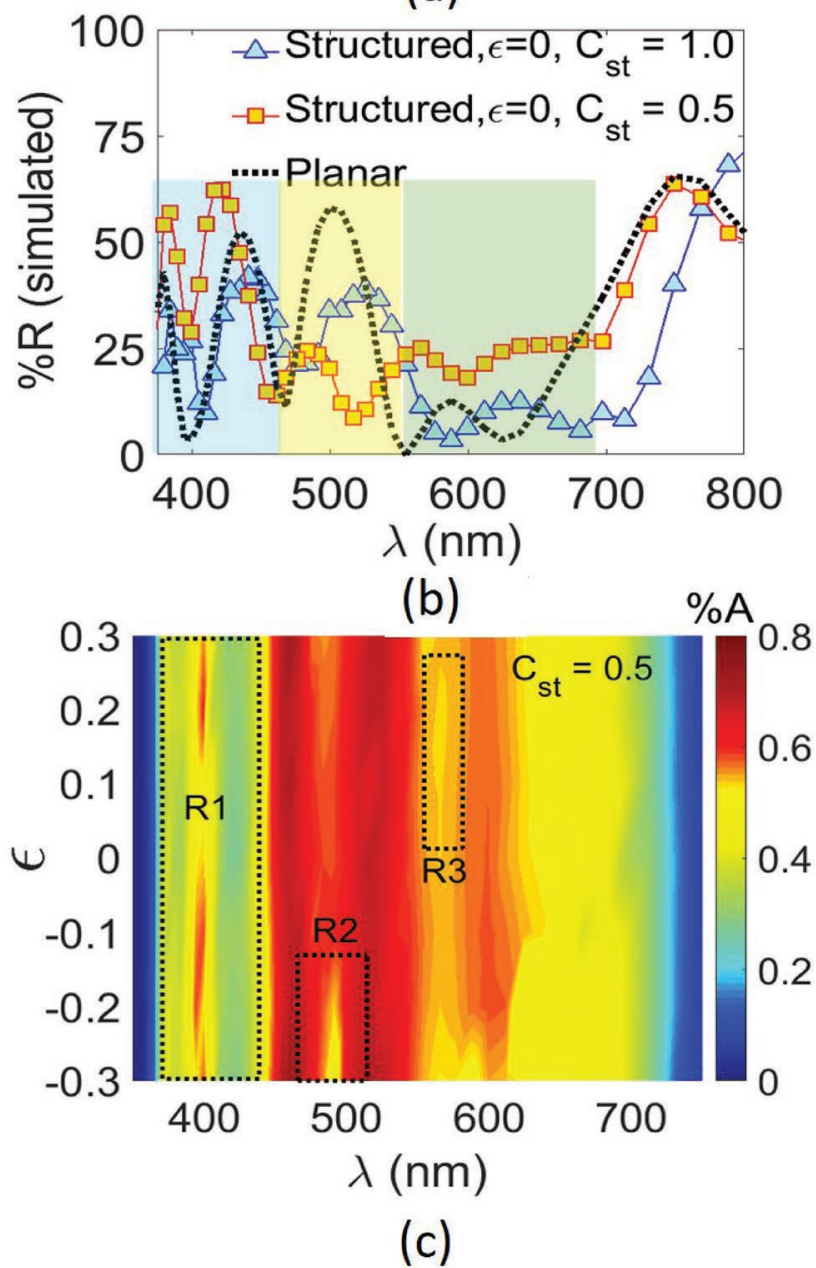

Figure 4. a) Simulated difference between the absorptances of the MW substrate incorporated unit cell and the average absorptance of the planar and completely nanostructured architectures. b) Simulated reflectance of the three devices. c) Simulated absorptance of an MW substrate incorporated device with $C_{\mathrm{st}}=0.5$ as a function of 1D strain applied to mold.

architecture ( $n=0$, in Figure 1$)$ and $A_{n=1}$ of the discontinuous nanostructured array $(n=1$, in Figure 1$)$. However, the absorptance, $A_{\text {multiscale }}$, of the device simulated with $50 \%$ coverage shows marked differences from the simulated linear combination (Figure 4a). The spectrum of the difference in the $A_{\text {multiscale }}$ and $A_{\text {superposed }}$ shows three distinct spectral windows $\mathrm{A}, \mathrm{B}$, and $\mathrm{C}$ as shown in Figure $4 \mathrm{a}$. In region $\mathrm{A}$, the multiscale structure results in a lesser absorptance than the constituent low-level structures. This is because of the resonant coupling of light entering from the structured domain into the planar domain. There is a subsequent increase in reflectance due to leakage from the waveguide (Figure $4 \mathrm{~b}$ ). In region $\mathrm{B}$, the absorptance of the multiscale architecture is higher than that of both the constituent structures corresponding to $n=0$ and $n=1$ (Figure 4a). This is because of weak coupling of light across the microdomains, due to wavelength-dependent nature of the cross-domain coupling.

This weak in-plane coupling possibly results in a larger portion of the light being coupled into the absorber, in the spectral window B, compared to the planar $(n=0)$ and completely nanostructured $(n=1)$ architectures. Consequently, a larger portion of the incident light is coupled to the active layer resulting ultimately in an improved absorptance in region B. This filtering of the incident light by the multiscale structure, which is unlike the effects produced by the planar and continuous nanostructured array, results in a more uniform absorptance spectrum as seen in Figure 1d, which also explains the improved spectral range of the devices. In region $\mathrm{C}$, there is a slightly reduced absorptance compared to the linear combination limit (Figure 4a). This is possibly due to weak coupling of scattered light into the planar microdomain and subsequent transport of the light out of the device laterally. The simulated spectral behavior in regions A and B clearly illustrates the role of the hierarchical structure. The resonant coupling of scattered light across microdomains, and the consequent changes in the reflectance and absorptance are the processes we refer to here as multiscale photonic phenomena, which are not seen in the continuum $(n=0)$ and the perfectly nanostructured $(n=1)$ architectures. This coupling of light across microdomains can be further visualized through the electric field patterns shown in Figure 1f. Whereas the incident wave has an electric field pointing only in the $x$-direction, the scattered wave patterns across the microdomains clearly show guided wave behavior with electric field modes having both $x$ and $y$ components. This conclusively proves the multiscale guided-wave behavior that underlies the improved spectral uniformity in the $\mathrm{MW}_{\varepsilon}$ architecture. This is a clear example that a hierarchical structure can exhibit photonic processes that are not noticeable in simpler defect-free structures and can be used to solve practical optoelectronic design problems.

\subsubsection{Effect of Strain}

The simulation of the effects of strain is quite complex because the actual sample would exhibit nanostructured periodic patterns with various parameters involved as seen in Figure 6b,c. Also, the simplified 2D model considered here does not consider the compression of the features in the in-plane direction normal to the applied strain at fabrication. However, a simplified 2D approximation of the problem is analyzed here, to obtain tensile and compressive effects. When a tensile stress is applied in the 
longitudinal direction (Figure 5d), there is compression in the axes perpendicular to the tensile direction. Atomic force microscopy (AFM) showed that there were no major changes in the height of the nanostructures (Figure 6a,b). However, in the inplane direction perpendicular to the direction of the applied tension, the nanostructures are compressed (Figure 6b) due to the Poisson's effect. Although both tensile and compressive features coexist on the same substrate, the simplified 2D model considered here cannot handle them simultaneously and thus the analyses of tensile and compressive features are separately considered. In reality, there will be interactions between these components and a more complex $3 \mathrm{D}$ geometry will be required to model these interactive nonlinear effects precisely. It should be noted here that the epoxy substrates themselves are not strained, but only the PDMS mold is strained while the epoxy is cast on it, thereby creating a change in the shape of the dome-like structure. There are distinct effects in the absorptance due to both tensile and compressive strains (Figure 4c). Region R1 marked on the lower wavelength side has a reduced absorptance, which was a consequence of the multiscale photonic transport that was explained in the previous section. Regions R2 and R3 show that there is a reduction in the absorptance in the mid-wavelength range (roughly $\lambda=475-600 \mathrm{~nm}$ ) due to compressed and elongated nanostructures, respectively. These effects explain the slight dip in the EQE of the device fabricated on the $\mathrm{MW}_{20}$ substrate. We further emphasize here that although a continuously nanostructured architecture $\left(C_{\mathrm{st}}=1.0\right)$ can exhibit tunability in its spectral response through application of strain fabrication, this tunability does not lead to an improved spectral uniformity, thus necessitating a combination of a multiscale structure and strain to obtain improved performances (see Section S8 in the Supporting Information). The actual light coupling processes in a real 3D architecture would be much more complex and will need extensive computational effort. However, from the perspective of design, this simplified approach shows that it is possible to tune the strain, at the time of fabrication, to simultaneously obtain optimal tensile and compressive effects, to effect a desired change in the optoelectronic spectrum. Use of mechanical strain as a design parameter would extend nanomolding and soft lithography beyond the conventional limits imposed by the geometry of the master template. This is the first step in the design of more complex multiscale structures that can offer novel avenues to finely control the flow of light, leading to many interesting applications in photonics and optoelectronics. Further, this effort also opens up avenues for theoretical exploration to study complex light transport through multiscale structures leading to novel solutions to light trapping in photovoltaic devices, which exceed the performance limits of current designs. Overall, we demonstrate a design-based, technologically viable self-assembly-based fabrication of hierarchically structured platforms for better performing photonic and optoelectronic devices.

\section{Conclusion}

This paper explores hierarchical multiscale patterning in the context of multiscale photonic processes. We identify the basis of the multiscale optical transport phenomena that do not manifest in the constituent lower length-scale structures and tap these coupled processes to solve the problem of spectral shaping in photodetectors. Particularly, we fabricated multiscale waveguides consisting of microscale clusters of planar and nanostructured subdomains and demonstrate that these waveguides can be used as substrates for optoelectronic spectral shaping in photodetectors. The use of multiscale structures leads to resonant coupling of scattered light from the nanostructured domains to the planar domains, a process that can occur only in a hierarchically structured architecture, consequently leading to suppression of the unwanted spectral features regarding optoelectronic performance. The nanostructures are further shaped using a novel mechanical strain applied during molding process through which the spectral uniformity of photodetectors built on these platforms was enhanced by nearly $10 \%$. The multiscale waveguide substrates fabricated with different strains are incorporated in photodetectors for simultaneously improving the spectral range and uniformity of the spectral band. This study shows possibilities to extend the limits of soft lithography beyond the limits of conventional molding and geometric parameters or resolution through the development of a mechanical-strain-assisted nanomolding process by which the optoelectronic spectra can be tuned. The experimental results supported by simulation results confirm the advantage of such unconventional approaches involving multiscale or hierarchical design and fabrication, and thus are expected to pave new avenues to solve optoelectronics-related engineering problems through hierarchical and multiscale structuring of materials.

\section{Experimental Section}

Multiscale Substrate Fabrication: Multiscale patterns were obtained using a soft-lithography-based two-step molding that has been demonstrated in an earlier work. ${ }^{[16]}$ With this technique, a monolayer of polystyrene nanospheres of diameter $400 \mathrm{~nm}$ was formed on a silicon substrate. Here, the fabrication process was further modified to tune the nanostructured features beyond the limits of the master template by applying mechanical strain. For this, the silicon substrate was placed at the center of a mold that resembled a standard tensile test specimen (Figure 5a) as per ASTM E8 standard.

The monolayer formed due to the nanospheres on silicon was discontinuous (Figure $5 \mathrm{~b}$ ) resulting in the formation of a network of nanostructured clusters interspersed with planar islands. The planar and the structured domains extended over a larger length scale ranging up to a few tens of micrometers. The domain sizes of the planar and the structured regions were further amenable to tuning through a simple variation of dilution parameters of the starting solution. ${ }^{[16]}$ PDMS was cast into this mold and cured at $65^{\circ} \mathrm{C}$ for $6 \mathrm{~h}$ in an oven. The PDMS was then peeled off and it held the inverse pattern of the monolayer, namely, a nanocup array network separated by the planar domains, embedded in the tensile specimen. The PDMS mold was then fixed across the two holders and a longitudinal tensile strain was applied as schematically shown in Figure $5 \mathrm{~d}$. Subsequently, the optically transparent substrates were fabricated at room temperatures by casting a transparent epoxy (Araldite LY5052, Huntsman) on the stretched PDMS mold, and allowing it to solidify over $24 \mathrm{~h}$, while keeping the mold stretched during the curing process. In addition to the multiscale pattern resembling that on the master template, the cured epoxy structures have multiperiodic nanostructured features, which arise as a result of using a strained intermediate mold. The details of the setup for applying controlled strains on the PDMS mold are given in Section S2 (Supporting Information).

Figure $6 a$ shows the hexagonal closed-packed 2D crystals $(d=400 \mathrm{~nm})$ having closed-packed nanosphere templated structures of 


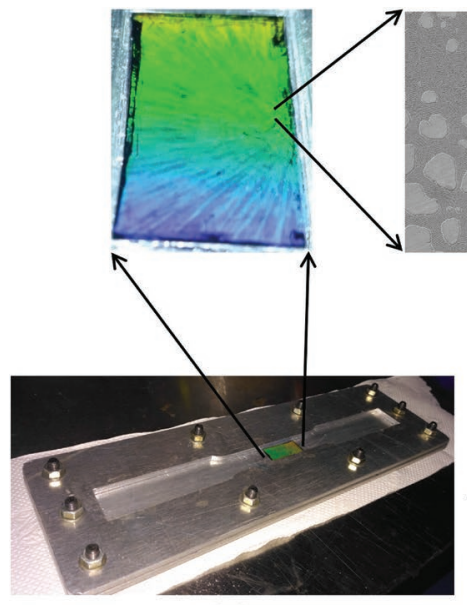

(a)

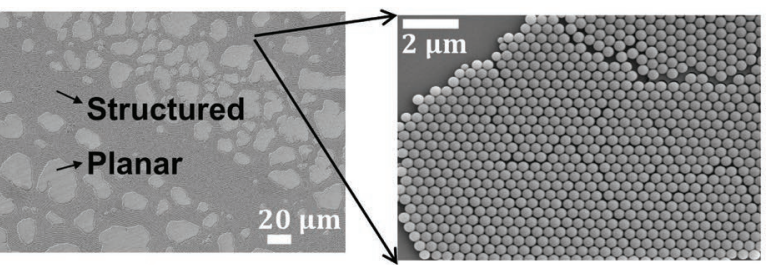

(b)

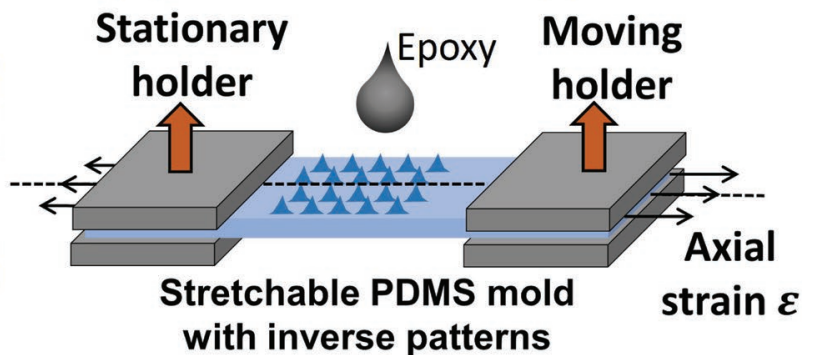

(d)

Figure 5. a) The multiscale silicon/polystyrene sphere master template, with a sphere diameter $w=400 \mathrm{~nm}$ placed in a mold based on tensile test specimen. b) Scanning electron microscopy image of the master template showing a discontinuous network of nanostructured regions interspersed with the planar regions. c) The nanostructured domain showing the hexagonally self-assembled nanospheres on the master template. d) Schematic of the inverse PDMS template with an applied strain on which the epoxy substrate material is cast under a constant strain applied to the mold.

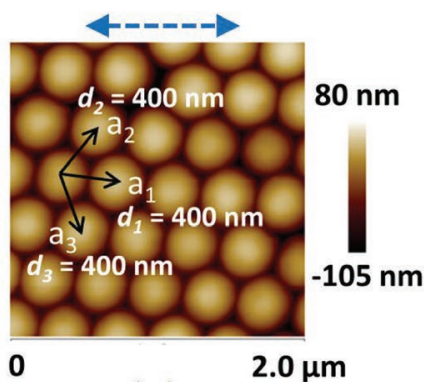

(a)

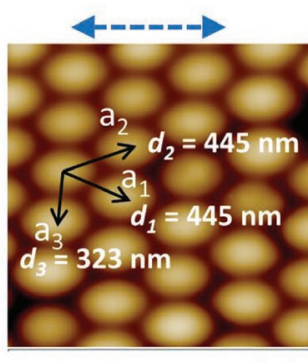

(b)

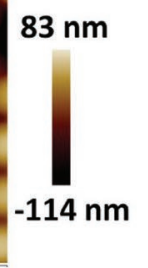

$2.0 \mu \mathrm{m}$

Planar domain

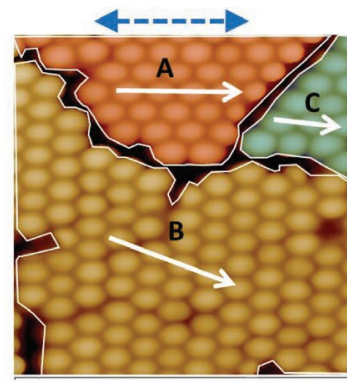

0

(c)
$5 \mu \mathrm{m}$

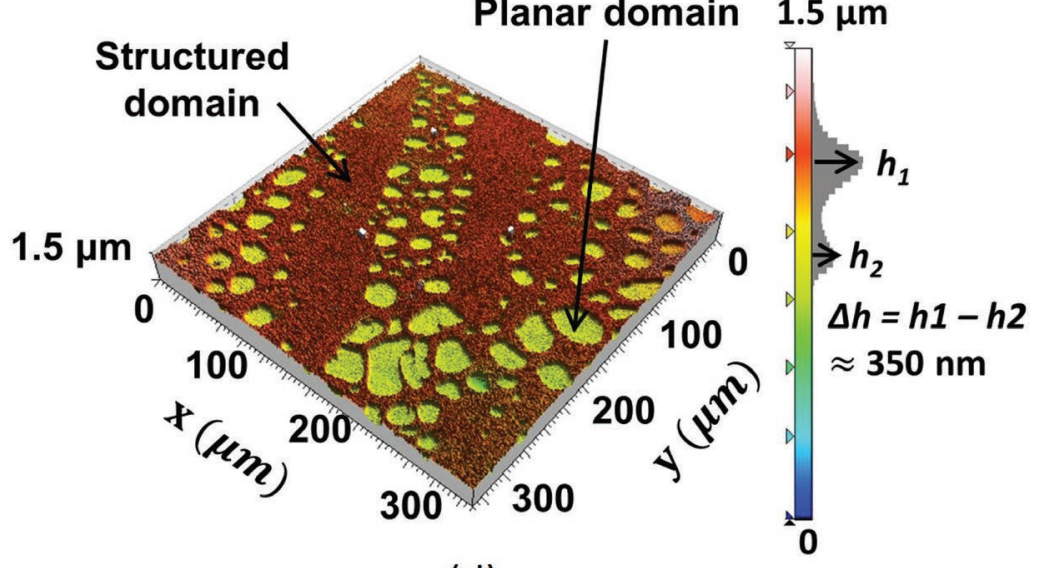

(d)

Figure 6. AFM images of the morphology of the nanostructure in the epoxy substrates fabricated with mechanical strains of a) $\varepsilon=0.0$ and b) $\varepsilon=0.2$ on the PDMS mold. c) AFM image illustrating the random orientation of crystallites within a substrate leading to a range of nanostructured periodicities across the various regions of the substrate. In (a)-(c), the blue dotted double-ended arrows on the top of the images indicate the approximate direction of the applied tensile strain (i.e., the reference $x$-axis). d) The large-area topography of the epoxy substrate showing a two-level structural hierarchy with an average height difference of $350 \mathrm{~nm}$ captured by an optical profilometer. 
nanostructured array in the finally formed epoxy substrate without any externally applied strain, although there could be residual strains due to curing of epoxy. Figure $6 \mathrm{~b}$ shows the morphology of a nanostructured crystallite fabricated with a strained mold, with $\varepsilon=0.2$. As seen here, the strained PDMS mold led to the breaking down of the monoperiodicity into three different lattice constants $d_{1}, d_{2}$, and, $d_{3}$.

Further, since each crystallite has a random in-plane orientation, within the defective 2D crystal in the master template, a strained mold led to a range of periodicities in terms of the lattice constants $d_{1}, d_{2}$, and $d_{3}$ (Figure $6 c$ ) across various regions in the sample. In Figure $6 c$, the crystallites $A, B$, and $C$ have different orientations and hence different lattice constants. At a larger scale, the 3D surface profile obtained using an optical profilometer revealed the formation of planar and nanostructured microscale domains on the epoxy, with an average height difference of roughly $350 \mathrm{~nm}$ between the domains, which was a little lesser than the diameter of the nanosphere (Figure $6 \mathrm{~d}$ ). This further verified the successful transfer of the multiscale pattern resulting from the discontinuous monolayer in the starting template. The fabrication method developed here can be extended to large values of strain, as demonstrated by the data in Section S3 (Supporting Information). The morphological details of the multiperiodic nanostructures on the epoxy for strains as large as $\varepsilon=40 \%$ are provided. Therefore, the strainassisted method developed here significantly extended the capabilities of self-assembly-based nanofabrication beyond the conventional limits of the monoperiodic master template.

Device Fabrication: A $300 \mathrm{~nm}$ thick $\mathrm{TiO}_{2}$ layer was deposited on the planar and multiscale patterned substrates that are referred to as $\mathrm{PW}$ and $\mathrm{MW}_{\varepsilon}$ substrates, respectively, where $\varepsilon$ in the subscript is the percentage strain applied on the PDMS mold during the fabrication of the epoxy substrate. In the context of the photodetector, the $\mathrm{TiO}_{2}$ film formed a high-index $(\approx 2.5)$ core of a dielectric waveguide that was sandwiched between the low-index substrate $(\approx 1.5)$ on one side and the anode $(\approx 1.5)$ on the other side.

Light can be confined within the waveguide through resonant scattering by the nanostructures in the $\mathrm{MW}_{\varepsilon}$ substrates, while in the PW an optical confinement can occur only through standing waves formed due to planar interface in the sandwich structure. Although any absorber material could be chosen for this purpose, an organic bulk heterojunction absorber was chosen, which can be easily solution processed at a relatively low temperature compared to inorganic materials. The device structure was as follows: $\mathrm{MW}_{\varepsilon} / \mathrm{PH} 1000(200 \mathrm{~nm}) /$ PEDOT:PSS( $\approx 50 \mathrm{~nm}) / \mathrm{PTB} \mathrm{P}: \mathrm{PC}_{71} \mathrm{BM}(150 \mathrm{~nm}) / \mathrm{Al}(100 \mathrm{~nm})$. The PH1000 layer was a solution-processed anode layer. This was spin coated over the waveguide substrate at $1000 \mathrm{rpm}$ for $60 \mathrm{~s}$. It yielded a sheet resistance of around $116 \Omega \mathrm{sq}^{-1}$.

PEDOT:PSS is a hole-selective layer that was spin coated over $\mathrm{PH} 1000$, at $5000 \mathrm{rpm}$ for $60 \mathrm{~s}$. Both these layers were individually annealed at $95{ }^{\circ} \mathrm{C}$ for 30 and $15 \mathrm{~min}$, respectively. The absorber was a bulk heterojunction formed between PTB7:PC ${ }_{70} \mathrm{BM}$ that was spin coated at $600 \mathrm{rpm}$ for $150 \mathrm{~s}$. The samples were further exposed to pressure of roughly $5 \times 10^{-6} \mathrm{mbar}$ for $3 \mathrm{~h}$ in a vacuum chamber following which an aluminum cathode layer of $100 \mathrm{~nm}$ thickness was deposited at a rate of roughly $1-3 \AA \mathrm{s}^{-1}$. The finished devices were characterized by their spectrally resolved optical and optoelectronic response in terms of their transmittance and reflectance spectra, and external quantum efficiency.

\section{Supporting Information}

Supporting Information is available from the Wiley Online Library or from the author.

\section{Acknowledgements}

The authors would like to acknowledge the support by the Ministry of Communication and Information Technology under a grant from the Centre of Excellence in Nanoelectronics, Phase II and MITO/EEC/ NKB/0096. The authors also thank the members of PV Lab at Center for Nano Science and Engineering, Indian Institute of Science, for helping with the deposition of $\mathrm{TiO}_{2}$ films.

\section{Conflict of Interest}

The authors declare no conflict of interest.

\section{Keywords}

hierarchical structures, light scattering, photodetectors, strain, waveguides

Received: March 15, 2019

Revised: May 15, 2019

Published online:

[1] R. Lakes, Nature 1993, 361, 511.

[2] K. Liu, L. Jiang, Nano Today 2011, 6, 155.

[3] K. B. Kancherla, D. B. Subbappa, S. R. Hiremath, B. Raju, D. R. Mahapatra, Composites, Part A 2019, 118, 131.

[4] X. Ren, R. Das, P. Tran, T. D. Ngo, Y. M. Xie, Smart Mater. Struct. 2018, 27, 023001.

[5] M. Liu, S. Wang, L. Jiang, Nat. Rev. Mater. 2017, 2, 17036.

[6] H. Butt, A. K. Yetisen, D. Mistry, S. A. Khan, M. U. Hassan, S. H. Yun, Adv. Opt. Mater. 2016, 4, 497.

[7] W.-K. Lee, J. Kang, K.-S. Chen, C. J. Engel, W.-B. Jung, D. Rhee, M. C. Hersam, T. W. Odom, Nano Lett. 2016, 16, 7121.

[8] K. Koch, B. Bhushan, Y. C. Jung, W. Barthlott, Soft Matter 2009, 5, 1386.

[9] W.-R. Wei, M.-L. Tsai, S.-T. Ho, S.-H. Tai, C.-R. Ho, S.-H. Tsai, C.-W. Liu, R.-J. Chung, J.-H. He, Nano Lett. 2013, 13, 3658.

[10] G. Yang, X. Li, Y. He, J. Ma, G. Ni, S. Zhou, Prog. Polym. Sci. 2018, $81,80$.

[11] L. Zhou, K. Zhang, Z. Hu, Z. Tao, L. Mai, Y. M. Kang, S. L. Chou, J. Chen, Adv. Energy Mater. 2018, 8, 1701415.

[12] G. D. Park, Y. C. Kang, Nano Res. 2018, 11, 1301.

[13] M. Lowman, Austral Ecol. 1986, 11, 163.

[14] C. Battaglia, C.-M. Hsu, K. Söderström, J. Escarré, F.-J. Haug, M. Charrière, M. Boccard, M. Despeisse, D. T. Alexander, M. Cantoni, ACS Nano 2012, 6, 2790.

[15] J. Zhu, C.-M. Hsu, Z. Yu, S. Fan, Y. Cui, Nano Lett. 2010, 10, 1979.

[16] A. K. Jagdish, K. Garg, P. C. Ramamurthy, D. R. Mahapatra, G. Hegde, Nanoscale 2018, 10, 3730.

[17] N. C. Linn, C.-H. Sun, P. Jiang, B. Jiang, Appl. Phys. Lett. 2007, 91, 101108.

[18] S. Mokkapati, K. Catchpole, J. Appl. Phys. 2012, 112, 101101.

[19] D.-H. Ko, J. R. Tumbleston, L. Zhang, S. Williams, J. M. DeSimone, R. Lopez, E. T. Samulski, Nano Lett. 2009, 9, 2742.

[20] K. Forberich, G. Dennler, M. C. Scharber, K. Hingerl, T. Fromherz, C. J. Brabec, Thin Solid Films 2008, 516, 7167.

[21] M. Niggemann, M. Riede, A. Gombert, K. Leo, Phys. Status Solidi A 2008, 205, 2862

[22] S.-F. Leung, K.-H. Tsui, Q. Lin, H. Huang, L. Lu, J.-M. Shieh, C.-H. Shen, C.-H. Hsu, Q. Zhang, D. Li, Energy Environ. Sci. 2014, 7, 3611.

[23] J. Yun, W. Wang, S. M. Kim, T.-S. Bae, S. Lee, D. Kim, G.-H. Lee, H.-S. Lee, M. Song, Energy Environ. Sci. 2015, 8, 932.

[24] H. Wang, D. Periyanagounder, A. Li, J. He, IEEE Access 2019, 7, 19395. 
[25] S. Das, M. J. Hossain, S.-F. Leung, A. Lenox, Y. Jung, K. Davis, J.-H. He, T. Roy, Nano Energy 2019, 58, 47.

[26] S. Gottheim, H. Zhang, A. O. Govorov, N. J. Halas, ACS Nano 2015, 9, 3284 .

[27] W. Tian, D. Liu, F. Cao, L. Li, Adv. Opt. Mater. 2017, 5, 1600468.

[28] L. Peng, L. Hu, X. Fang, Adv. Funct. Mater. 2014, 24, 2591.

[29] Q. Zhang, G. Cao, J. Mater. Chem. 2011, 21, 6769.

[30] D. Hwang, J.-S. Jin, H. Lee, H.-J. Kim, H. Chung, D. Y. Kim, S.-Y. Jang, D. Kim, Sci. Rep. 2015, 4, 7353.

[31] Y. Xia, G. M. Whitesides, Annu. Rev. Mater. Sci. 1998, 28, 153.

[32] J. C. Hulteen, R. P. Van Duyne, J. Vac. Sci. Technol., A 1995, 13, 1553.

[33] X. Liang, R. Dong, J. C. Ho, Adv. Mater. Technol. 2019, 4, 1800541.

[34] J. R. D. Retamal, C.-H. Ho, K.-T. Tsai, J.-J. Ke, J.-H. He, IEEE Trans. Electron Devices 2019, 66, 938.

[35] Y. Ai, T. H. Hsu, D. C. Wu, L. Lee, J. Chen, Y. Chen, S. Wu, C. Wu, Z. M. Wang, Y. Chueh, J. Mater. Chem. C 2018, 6, 5514.

[36] S. Y. Chou, P. R. Krauss, P. J. Renstrom, J. Vac. Sci. Technol., B: Microelectron. Nanometer Struct.-Process., Meas., Phenom. 1996, $14,4129$.
[37] J.-Y. Shiu, C.-W. Kuo, P. Chen, C.-Y. Mou, Chem. Mater. 2004, 16, 561.

[38] C. L. Haynes, R. P. Van Duyne, J. Phys. Chem. B 2001, 105, 5599.

[39] M. A. Green, A. Ho-Baillie, H. J. Snaith, Nat. Photonics 2014, 8, 506.

[40] A. Hagfeldt, G. Boschloo, L. Sun, L. Kloo, H. Pettersson, Chem. Rev. 2010, 110, 6595 .

[41] H. Hoppe, N. S. Sariciftci, in Photoresponsive Polymers II, Springer, Berlin 2007, p. 1.

[42] X. Gong, M.-H. Tong, S. H. Park, M. Liu, A. Jen, A. J. Heeger, Sensors 2010, 10, 6488

[43] X. Gong, M. Tong, Y. Xia, W. Cai, J. S. Moon, Y. Cao, G. Yu, C.-L. Shieh, B. Nilsson, A. J. Heeger, Science 2009, 325, 1665.

[44] K. Kishino, M. S. Unlu, J. I. Chyi, J. Reed, L. Arsenault, H. Morkoc, IEEEJ. Quantum Electron. 1991, 27, 2025.

[45] N. P. Sergeant, A. Hadipour, B. Niesen, D. Cheyns, P. Heremans, P. Peumans, B. P. Rand, Adv. Mater. 2012, 24, 728.

[46] K. H. An, B. O'Connor, K. P. Pipe, M. Shtein, Org. Electron. 2009, 10, 1152. 